\title{
Radiation Pattern of an UWB Wearable Antenna: A Preliminary Study
}

\author{
Wen-Bin Yang, Kamran Sayrafian-Pour \\ Information Technology Laboratory \\ National Institute of Standards and Technology \\ USA \\ \{wyang, ksayrafian\}@nist.gov
}

\begin{abstract}
Inhomogeneity of the propagation medium in body area networks could have a great impact on the sensor node's antenna characteristics. This in turn could affect the quality of the communication links unless the impact of the human body has been properly considered during the design phase of the antenna. In this paper, we investigate the impact of the antenna's placement and its distance from the body surface on its radiation pattern. It is shown that the antenna placement could affect the inbody radiation pattern of the antenna.
\end{abstract}

\section{Keywords}

Antenna radiation pattern; Specific absorption rate; Body area networks

\section{INTRODUCTION}

Body Area Networks (BANs) consist of wearable or implantable sensors that can establish two-way wireless communication with a controller node that could be either worn or located in the vicinity of the body $[1,7,8]$. Inhomogeneity of the propagation medium (i.e. human body) in these networks could have a great impact on the sensor node's antenna characteristics. This in turn could affect the quality of the communication links unless the impact of the human body has been properly considered during the design phase of the antenna. For wearable sensors nodes, the antenna's adjacency to the body, and more precisely its location (e.g., head, chest, waist, and left wrist) and distance to the body surface can affect the antenna's radiation pattern, radiation efficiency, and resonance frequency. This could directly impact communication link quality between the sensors and the controller node in body area networks.

By calculating the radiation pattern at various distances separating the antenna and the human body surface, a statistical analysis of the impact of the human body on the radiation pattern of wearable antennas operating at $2.4 \mathrm{GHz}$ was done in [2]. If the sensor (and therefore the antenna) is not directly attached to the body, the distance (i.e. separation) between the antenna and the body surface may change significantly as the user moves or changes posture [9]. In [2], it was assumed that the statistical distribution of this gap is known (e.g. Uniform or Rayleigh).

In this paper, instead of taking a statistical approach, we consider the case where the wearable sensor is directly located on the body surface. We investigate the change in antenna radiation pattern for various distances from the body surface.
The remainder of this paper is organized as follows. Section II describes the human body model and antenna parameters. Section III focuses on the analysis of antenna radiation pattern. SAR analysis is presented in Section IV. Simulation results and conclusions are provided in Sections V and VI respectively.

\section{SYSTEM DESCRIPTION}

\subsection{Human Body Model}

In our analysis, a sophisticated male Human Body Model (HBM) with over 300 parts has been used (see Fig. 1). The dielectric properties of each part in the model are frequency dependent. They can also be modified by the user, if custom changes are desired. Two levels of resolutions can be employed when using the model, i.e., 2 and $4 \mathrm{~mm}$. We have also used $\mathrm{HFSS}^{1}$ as the propagation engine to compute a variety of different electromagnetic quantities such as the magnitude of electric and magnetic fields; antenna parameters such as intensity, gain, radiation power, and radiation efficiency.

\subsection{Wearable Antenna}

Antenna is a key component of wireless body area networks. There exist many technical challenges in designing appropriate antennas for BAN. Biocompatibility and size limitation from one side and bandwidth, efficiency and gain within the limited antenna volume are among the challenges for such antennas. When designing these antennas, the influence of the human body on their characteristics (e.g., gain pattern distortion) has to be carefully accounted for; otherwise, performance degradations such as pattern fragmentation, power absorption, and central frequency shift will occur [3, 4]. As UWB technology seems to be a favorable candidate for wearable BAN applications, in this study,

we have used a UWB loop antenna as shown in Fig. 2. This antenna, which has dimensions of $29.25 \times 38.5 \times 1 \mathrm{~mm}$, has an operating frequency range of $3.1-5.1 \mathrm{GHz}$. It is printed on a side of FR4 substrate with dielectric constant of $\varepsilon_{\mathrm{r}}=4.4$ and loss tangent of $\tan \theta=0.02$.

\footnotetext{
${ }^{1}$ HFSS is a registered trademark of ANSYS, Inc. The HFSS has been used in this research to foster understanding. Such identification does not imply recommendation or endorsement by the National Institute of Standard and Technology, nor does it imply that this product is necessarily the best available for the purpose.
} 


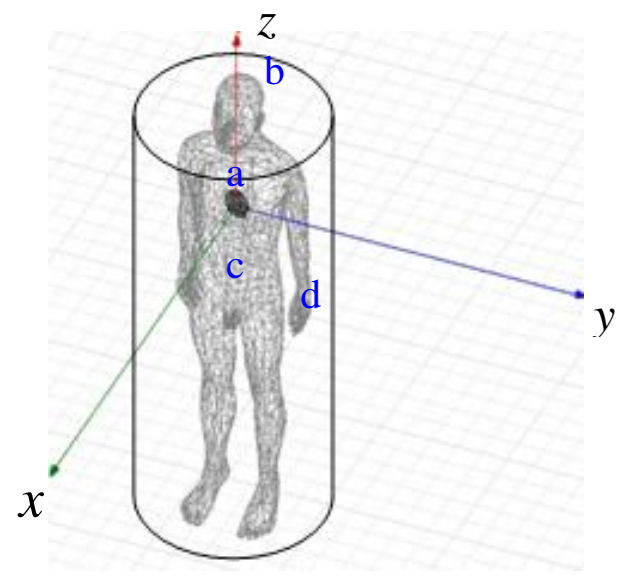

Figure 1: Human body model

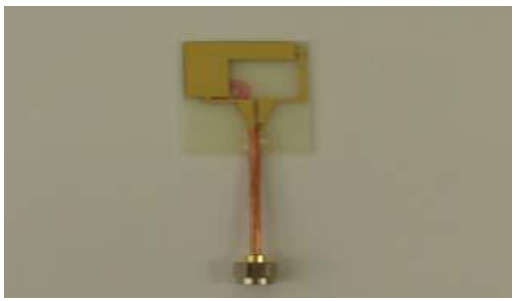

Figure 2. The UWB Loop Antenna

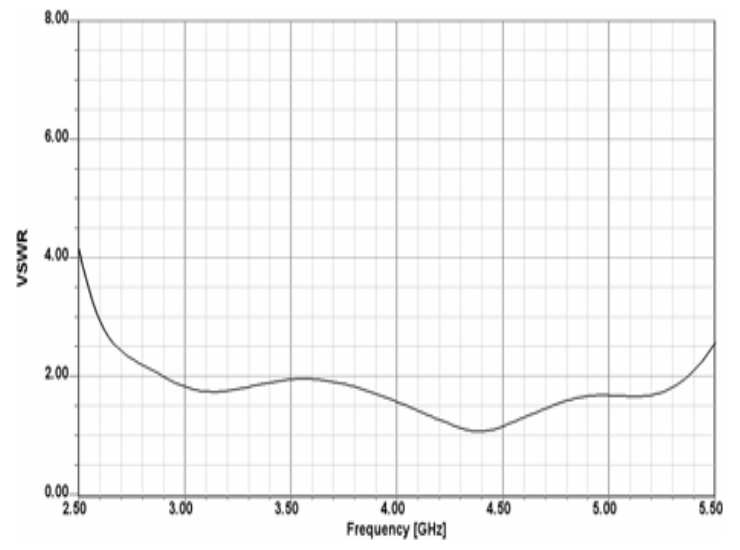

Figure 3. The UWB antenna VSWR in proximity to the body surface

Figure 3 displays the VSWR (Voltage Standing Wave Ratio) of the antenna in proximity to the human body. Detailed study of the characteristics of this antenna in free space and in direct contact with the human body has been provided in [5].

\section{ANTENNA RADIATION PATTERN}

In some BAN applications (and based on the particular node design and its packaging), the antenna might be located on the body surface. As dielectric properties of various parts of the body (e.g. left wrist, chest, waist, or head) could be substantially different, the resulting radiation pattern of the antenna could depend on its actual location on the body surface. In addition, the gap between the antenna and body surface, and the type of the material used in between are among other factors that could impact its radiation pattern.

The antenna radiation pattern is defined as the gain $G(\theta, \phi)$ in a given spherical coordinate direction $(\theta, \phi)$. The radiation intensity, $U(\theta, \phi)$, is also defined as the power radiated from an antenna per unit solid angle (in steradian). It can be described by the following equation [6]:

$$
U(\theta, \phi)=\frac{1}{2} \frac{r^{2}|E|^{2}}{\eta_{0}}
$$

Where

$|E|$ is the magnitude of the E-field,

$\eta_{0}$ is the intrinsic impedance of free space,

$r$ is the distance from the antenna.

Note that radiation intensity is independent of $r$. Antenna gain is $4 \pi$ times the ratio of antenna's radiation intensity in a given direction to the total power accepted by the antenna, i.e.

$$
G(\theta, \phi)=\frac{4 \pi U(\theta, \phi)}{P_{a c c}}
$$

Where $P_{a c c}$ is the accepted power in watts (i.e. time-averaged power entering the antenna structure).

\section{SIMULATION RESULTS}

In our simulations, we have analyzed the influence of the human body on antenna radiation pattern as well as the resulting SAR for the following scenarios: (i) UWB antenna placed on front waist, left wrist, head, and chest, (ii) UWB antenna placed at varying distances above body surface, and (iii) Using human body model with the $2 \mathrm{~mm}$ and $4 \mathrm{~mm}$ resolutions. The antenna radiation pattern is for the $x-y$ plane (i.e. parallel to the ground) as shown in Fig. 1.

Figure 4 shows the UWB antenna pattern when it is located on the waist, left wrist, head, and chest. In all these locations, the antenna was placed $10 \mathrm{~mm}$ above the body surface and the $2 \mathrm{~mm}$ body model was used. For comparison, the antenna pattern without the human body is also plotted in this figure. The influence of the human body on the radiation pattern (and most importantly its dependency on the exact antenna location on the body) is clearly observed. As a result of the body influence, the peak may vary by almost $9.4 \mathrm{~dB}$. Also, the gain in the direction pointed toward the human body may vary by more than $15 \mathrm{~dB}$. The influence of the human body seems to be less at the left wrist position; and therefore, the deviation of the gain pattern compared to the isolated antenna case is less than other locations.

At the same time, looking at the antenna pattern in the direction away from the human body, no significant difference is observed for all locations considered in our simulations. Therefore, the communication link from wearable antenna to a node outside and 
away from the human body seems to be relatively independent of the wearable antenna whereabouts on the body surface.

The antenna radiation patterns at the chest, head, front waist, and left wrist for various distances from the body surface are shown in Figures 5-8, respectively. For these simulations, the antenna separation varied from 0 to $20 \mathrm{~mm}$ with a step size of $5 \mathrm{~mm}$. The $2 \mathrm{~mm}$ resolution in the body model was used to obtain these results. When the antenna is located on the chest, its radiation patterns do not exhibit any significant differences for all 5 distances (see Fig. 5). However, the influence of the human body is quite evident when considering the antenna pattern in isolation. The antenna pattern distance dependency for the head position (Fig. 6) is more pronounced compared to the chest position. In addition, due to the high attenuation of the skull, the values of the antenna gains inside the body are much smaller compared to other locations considered in this study.

The gain patterns when the antenna is located on the front waist are shown in Fig. 7. When the antenna is directly placed on the body surface (i.e. $0 \mathrm{~mm}$ gap), a significant impact of the human body is observed in both directions, i.e., toward and away from the body. Looking at the gain values inside the body, the distance dependency of the antenna pattern is clearly observed for this scenario. For the left wrist position, the pattern is less influenced by the body as shown in Fig. 8. This could be expected since the bulk of the human body is away from the antenna when it is placed on the left wrist.

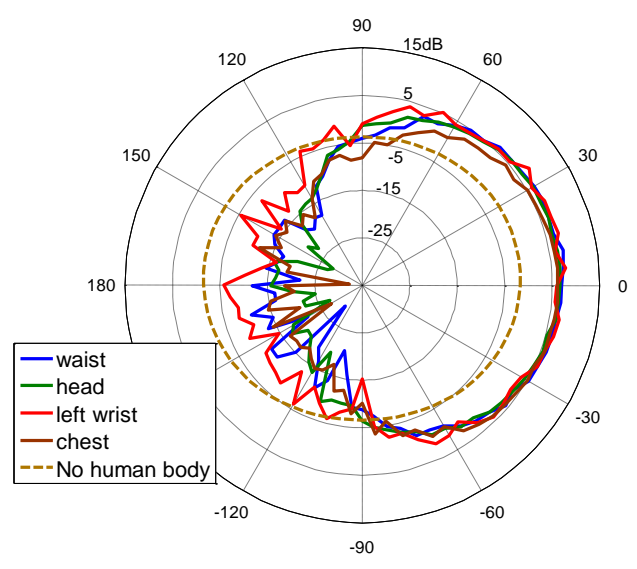

Figure 4: UWB antenna pattern vs. locations (10mm above body surface)

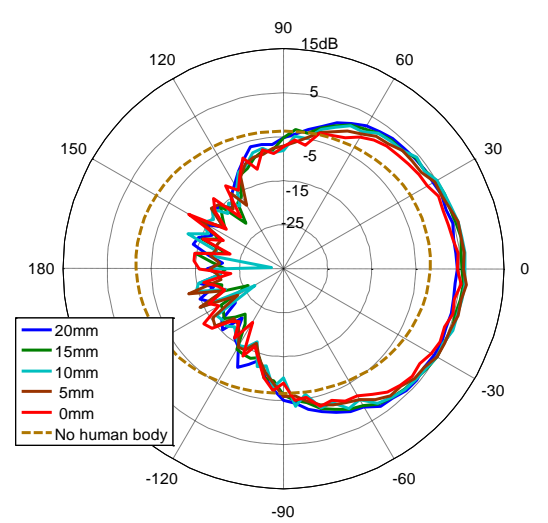

Figure 5: UWB antenna pattern vs. distance (chest)

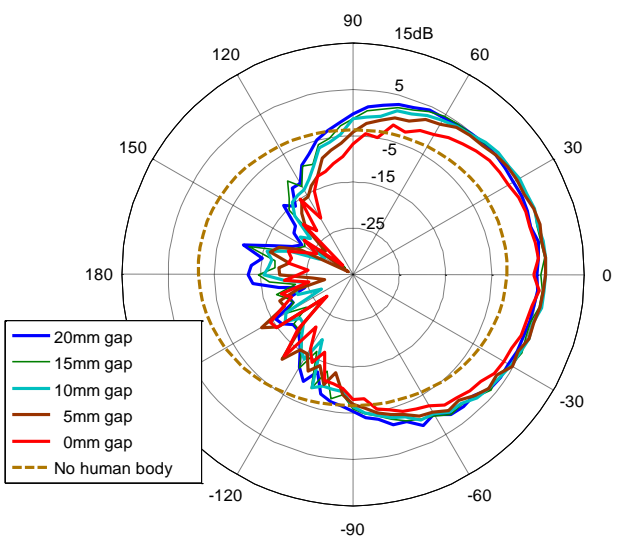

Figure 6: UWB antenna pattern vs. distance (head)

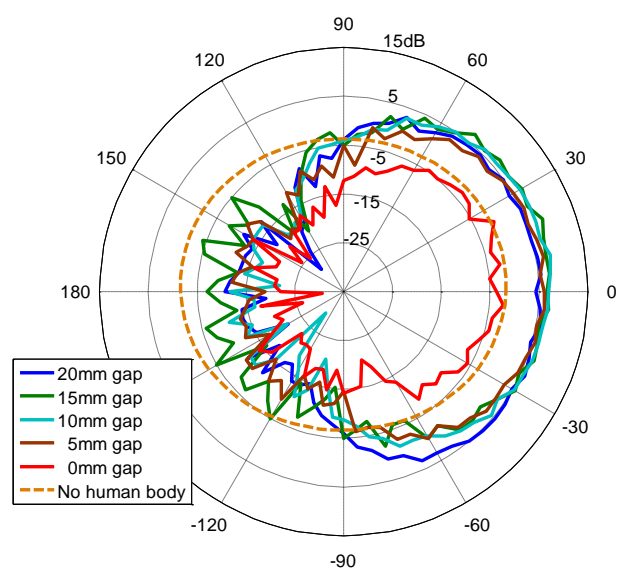

Figure 7: UWB antenna pattern vs. distance (front waist) 


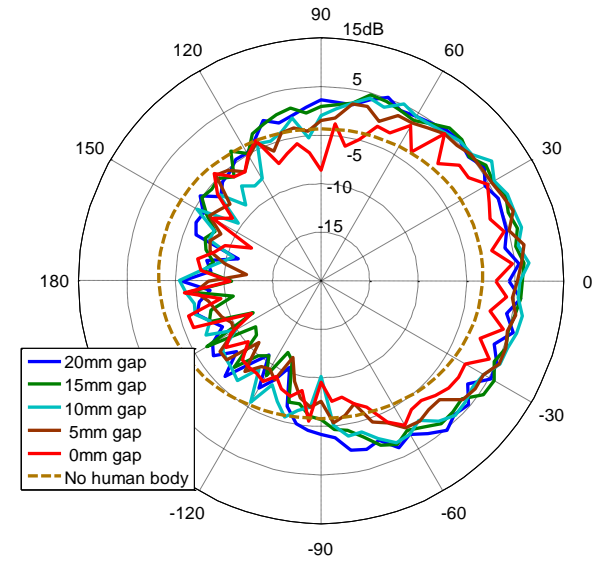

Figure 8: UWB antenna pattern vs. distance (left wrist)

\section{CONCLUSIONS}

The influence of the human body on the radiation pattern of a wearable antenna for different antenna locations on the body surface were studied in this paper. Other than the location of the antenna, other factors considered in our study were antenna separation from the body surface ( 0 to $20 \mathrm{~mm}$ range). The antenna gain pattern depends on the exact location and distance of the antenna from the body. Intelligent antenna design is the key to establish communication links with adequate range and acceptable quality. Other frequencies of interest for BAN wearable applications, e.g., $2.4 \mathrm{GHz}$ ISM band, can also be studied using the same methodology outlined in this paper. The authors plan to continue this work and also investigate SAR issues for wearable node communication. Further experimental studies and comparison with phantom models are required to validate the results obtained here.

\section{REFERENCES}

[1] G. Yang, "Body sensor networks", Springer-Verlag London Limited 2006, ISBN 1-84628-272-1.

[2] M. Mackowiak, et al, "A statistical analysis of the influence of the human body on the radiation pattern of wearable antennas", IEEE PIMRC 2011, pp. 2224-2228, Toronto, Canada, September 2011.

[3] K. Y. Yazdandoost, R. Kohno, "UWB Antenna for Wireless Body Area Network", Proceedings of Asia-Pacific Microwave Conference 2006.

[4] P. S. Hall, Y. Hao, "Antennas and propagation for body centric wireless communications", Artech House, Inc. 2006, ISBN 1-58053-493-7.

[5] K. Y. Yazdandoost, R. Kohno, "Ultra Wideband L-loop antenna," IEEE International Conference on UltraWideband, 2005.

[6] E.C. Jordan and K.G. Balmain, "Electromagnetic Wave and Radiating Systems", Prentice-Hall, Inc., 1968.

[7] W.-B. Yang, K. Sayrafian-Pour, J. Hagedorn, J. Terrill, and K. Y. Yazdandoost, "Simulation Study of Body Surface RF Propagation for UWB Wearable Medical Sensors", Proceedings of the 2nd International Symposium on Applied Sciences in Biomedical and Communication Technologies, IEEE ISABEL 2009, Slovakia, Nov. 2009.

[8] A. Fort, J. Ryckaert, C. Desset, P. Doncker, P. Wambacq, L. V. Biesen, "Ultra-Wideband Channel Model for Communication Around the Human Body", IEEE Journal on Selected Areas in Communications, Vol. 24, No. 4, April 2006.

[9] M. Quwaider, J. Rao and S. Biswas, "Body-Posture-Based Dynamic Link Power Control in Wearable Sensor Networks", IEEE Communications Magazine, pp. 134-142, July 2010.

[10] C. Polk, E. Postow, "Biological effects of electromagnetic fields”, CRC Press, Inc., 1996, ISBN 0-8493-0641-8. 